\title{
Knockdown of IncRNA SNHG14 alleviates LPS-induced inflammation and apoptosis of PC12 cells by regulating miR-181b-5p
}

\author{
HUI JIANG ${ }^{*}, \mathrm{JIE} \mathrm{NI}^{2 *}, \mathrm{YAN}^{2} \mathrm{HENG}^{3}$ and YUN XU ${ }^{1}$ \\ ${ }^{1}$ Department of Neurology, Jiangsu Province Hospital of Chinese Medicine, Affiliated Hospital \\ of Nanjing University of Chinese Medicine; ${ }^{2}$ Department of Emergency, Affiliated Drum \\ Tower Hospital of Nanjing University; ${ }^{3}$ Department of Neurology, Affiliated Hospital of \\ Nanjing University of Chinese Medicine, Nanjing, Jiangsu 210000, P.R. China
}

Received August 24, 2020; Accepted December 8, 2020

DOI: $10.3892 /$ etm.2021.9928

\begin{abstract}
Spinal cord injury (SCI) is a traumatic central nervous system disorder that leads to permanent functional loss, and unavailable treatment of this disease results in poor quality of life. However, the specific role of long non-coding RNA small nucleolar RNA host gene 14 (lncRNA SNHG14) in SCI has not been fully studied. The aim of the current study was to investigate the role of SNHG14 and its regulatory mechanism in lipopolysaccharide (LPS)-induced PC-12 cells. LPS was used to stimulate PC-12 cells to simulate inflammatory injury following SCI in vitro. Cell viability and apoptosis were respectively assessed by Cell Counting Kit-8 assay and TUNEL assay. Western blotting was performed to detect the expressions of apoptosis-related proteins. The mRNA levels of SNHG14 and microRNA (miR)-181b-5p were detected by reverse transcription-quantitative PCR. The target of SNGH14 was predicted by bioinformatics analysis and subsequently validated by a luciferase reporter assay. ELISA was then used to detect the levels of inflammatory factors. The results indicated that LPS induced inflammation, decreased cell viability and increased the apoptosis of PC-12 cells. Interference of SNHG14 alleviated this type of injury of PC-12 cells. Bioinformatics prediction and luciferase reporter assay demonstrated that miR-181b-5p could directly bind to SNHG14. Moreover, mechanistic investigations revealed that the miR-181b-5p inhibitor could reverse the inhibitory effects
\end{abstract}

Correspondence to: Dr Yun Xu, Department of Neurology, Jiangsu Province Hospital of Chinese Medicine, Affiliated Hospital of Nanjing University of Chinese Medicine, 321 Zhongshan Road, Gulou, Nanjing, Jiangsu 210000, P.R. China

E-mail: xuyunnanjing@163.com

${ }^{*}$ Contributed equally

Key words: long non-coding RNA small nucleolar RNA host gene 14, inflammation, apoptosis lipopolysaccharide, microRNA-181b-5p of SNHG14 silencing on cell viability, inflammation and apoptosis of PC-12 cells. To conclude, the present results showed that SNHG14 knockdown alleviated PC-12 cell inflammation and apoptosis induced by LPS via regulating miR-181b-5p, which might provide a novel insight into the treatment of SCI.

\section{Introduction}

The spinal cord, which functions as a transporter to relay messages from the brain to the body and vice versa, transmits messages from the autonomic nervous system, which regulates numerous physiological functions, including heartbeat, blood pressure and temperature regulation. However, damage to the spinal cord can perturb all these functions. Spinal cord injury (SCI) is a severe central nervous system disorder that has been increasingly observed in the clinic over the past few years in China (1). Due to lack of successful treatment for SCI, 50-80\% of patients with SCI suffer with long-term moderate to severe traumatic pain, which significantly impacts the families of the patients both financially and in providing care (2).

Since significant damage is produced by secondary injury alone, the inhibition of processes involved in secondary injury may be one of the most effective approaches to prevent the deterioration of SCI and promote functional recovery $(3,4)$. The secretion of stimulating cytokines, which can facilitate neuronal damage and cell apoptosis, triggers the stimulus response and secondary injury. Previous studies have demonstrated that the levels of inflammatory factors, including IL-1 $\beta$, TNF- $\alpha$ and IL-6, were elevated at the early SCI stage (5-7). Interestingly, inhibiting the secretion of these inflammatory factors was reported to serve a central role in protecting nerve cells (6). Thus, investigating the molecular mechanism by which inflammatory cytokines could be inhibited may be a novel and effective strategy for the treatment of SCI.

Long non-coding RNAs (lncRNAs) are non-coding RNAs of $>200$ nucleotides in length that lack protein-coding ability (8). LncRNAs were reported to be associated with the initiation and progression of numerous types of diseases, including Parkinson's disease, leukemia and periodontitis (9-13). Knockdown of small nucleolar RNA host gene 14 
(SNHG14) protected nerve cells from damage by alleviating inflammation (14). In addition, IncRNA SNHG14 was revealed to promote neuronal impairment and inflammatory response induced by cerebral ischemia/reperfusion injury by regulating the microRNA (miRNA/miR)-136-5p/Rho-associated protein kinase 1 (ROCK1) signaling pathway, while SNHG14 knockdown could improve neurological function in vivo (15). SNHG14 knockdown was also discovered to alleviate lipopolysaccharide (LPS)-induced acute lung injury through the miR-34c-3p-dependent inhibition of Wnt inducible signaling pathway protein 1 (16).

miRNAs are a class of non-coding RNAs that serve as important gene expression regulators (17). miRNAs negatively regulate target gene expression at the posttranscriptional level, which in turn regulates numerous cellular processes, such as cell differentiation, aging and apoptosis $(17,18)$. Previous studies have reported that miR-181a/b-5p, which was found to modulate vascular inflammation and endotoxin tolerance, alleviated inflammation in monocrotaline-induced pulmonary arterial hypertension by targeting endocan $(19,20)$.

To the best of our knowledge, no previous studies have reported the role of SNHG14 in SCI. Therefore, the present study aimed to investigate whether SNHG14 could participate in the neuronal protection and alleviate inflammation by regulating miR-181a/b-5p in SCI, in addition to determining the underlying mechanism.

\section{Materials and methods}

Cell culture and treatment. The PC-12 cell line was purchased from The Cell Bank of Type Culture Collection of the Chinese Academy of Sciences. The cells were cultured at a density of $1 \times 10^{4}$ cells/ml in DMEM-high glucose (Sigma-Aldrich; Merck KGaA) supplemented with 10\% FBS (Gibco; Thermo Fisher Scientific, Inc.) and $100 \mathrm{U} / \mathrm{ml}$ penicillin/streptomycin (Gibco; Thermo Fisher Scientific, Inc.). The cells were maintained in a humidified incubator with $5 \% \mathrm{CO}_{2}$ at $37^{\circ} \mathrm{C}$ and the medium was replaced every 2-3 days.

To stimulate inflammatory cell injury following SCI, PC-12 cells were treated with 1, 2,5 or $10 \mu \mathrm{g} / \mathrm{ml}$ LPS (Sigma-Aldrich; Merck KGA) for $12 \mathrm{~h}$. In addition, the 12-h LPS treatment of PC-12 cells was conducted after transfection.

Cell transfection. Short hairpin RNA (shRNA) targeting SNHG14, vector, miR-181b-5p mimic, miR-181b-5p inhibitor and their corresponding negative controls (NCs) were purchased from Shanghai GenePharma Co., Ltd. miR181b-5p mimic, 5'-AACAUUCAUUGCUGUCGGUGGGCA CCGACAGCAAUGAAUGUUUU-3'; mimic NC, 5'-UUC UCCGAACGUGUCACGUTTACGUGACACGUUCGGAGA ATT-3'; miR-181b-5p inhibitor, 5'-CCCACCGACAGCAAU GAAUGUU-3' and Inhibitor NC: 5'-UCACAACCUCCUAGA AAGAGUAGA-3'. A total of $80 \mathrm{nM}$ shRNA-SNHG14/shRNA$\mathrm{NC}, 50 \mathrm{nM} \mathrm{miR}-181 \mathrm{~b}-5 \mathrm{p} \mathrm{mimic} / \mathrm{mimic}-\mathrm{NC}$ and $50 \mathrm{nM}$ miR-181b-5p inhibitor/inhibitor-NC were transfected into PC12 cells at $80 \%$ confluence using Lipofectamine ${ }^{\circledR} 2000$ reagent (Invitrogen; Thermo Fisher Scientific, Inc.). Following $48 \mathrm{~h}$ of transfection, the cells were collected for subsequent experiments.
Cell Counting Kit-8 (CCK-8) assay. The cells were seeded into 96-well plates at a density of $1 \times 10^{4}$ cells/well and stimulated with LPS at $37^{\circ} \mathrm{C}$ for $12 \mathrm{~h}$. Following stimulation, $10 \mu \mathrm{l}$ CCK-8 reagent (Dojindo Molecular Technologies, Inc.) was added into the medium and incubated at $37^{\circ} \mathrm{C}$ for a further $2 \mathrm{~h}$. Finally, the absorbance of the cells was determined using a spectrophotometer at the wavelength of $450 \mathrm{~nm}$.

TUNEL assay. A TUNEL detection kit (OriGene Technologies, Inc.) was used to analyze the apoptosis of PC12 cells following LPS induction. A six-well plate was added with a small amount of culture medium $(500 \mu \mathrm{l})$ in order for the slides to be in close contact with the plate. Coverslips were then carefully placed in the plate. Following indicated treatments, PC-12 cell suspension was added to the coverslip at a density of $1 \times 10^{5}$ cells/well, which was incubated at $37^{\circ} \mathrm{C}$ in a $5 \% \mathrm{CO}_{2}$ chamber. The coverslips were washed in $37^{\circ} \mathrm{C}$ PBS three times for 3-5 sec each time, fixed in $4 \%$ paraformaldehyde for $15 \mathrm{~min}$ at room temperature and then cleaned with $37^{\circ} \mathrm{C}$ deionized water. The coverslips were dried on filter paper and glued to the slides with neutral balsam mounting medium. The sample slide was digested with protease $\mathrm{K}$ and then treated with terminal deoxynucleotidyl transferase and biotin-dUTP at $37^{\circ} \mathrm{C}$ for $60 \mathrm{~min}$. The slides were then sealed with sealing liquid and incubated with streptavidin-horseradish peroxidase (HRP) working liquid (cat. no. AR100017; OriGene Technologies, Inc.) and 3,3-diaminobenzidine color reagent (OriGene Technologies, Inc.). Cell nucleus were stained with hematoxylin at room temperature for $5 \mathrm{~min}$ and washed by PBS for three times. Finally, the color was observed and apoptotic cells were counted using a light microscope (magnification, x200) from five fields of view.

Reverse transcription-quantitative PCR (RT-qPCR). Total RNA from PC-12 cells was extracted and purified using TRIzol $^{\circledR}$ reagent (Invitrogen; Thermo Fisher Scientific, Inc.). Total RNA was reverse transcribed into cDNA using a PrimeScript $^{\mathrm{TM}}$ II 1st Strand cDNA Synthesis kit (Takara Bio, Inc.) using the following protocol: $65^{\circ} \mathrm{C}$ for $10 \mathrm{~min}, 25^{\circ} \mathrm{C}$ for $10 \mathrm{~min}, 37^{\circ} \mathrm{C}$ for $60 \mathrm{~min}$ and $70^{\circ} \mathrm{C}$ for $10 \mathrm{~min}$. qPCR was subsequently performed on an ABI Prism 7500 Sequence Detection system (Applied Biosystems; Thermo Fisher Scientific, Inc.) with a SYBR Green Real-Time PCR kit (Takara Bio, Inc.). The PCR reaction mixture $(20 \mu \mathrm{l})$ consisted of $10 \mu \mathrm{l}$ Real Time PCR Master mix, $2 \mu \mathrm{l}$ primer mix $(0.2 \mu \mathrm{M})$ and $20 \mathrm{ng}$ cDNA diluted in RNase-free water. The primer sequences were as follows: SNHG14 forward, 5'-CGTTGTCGAAAGCTAAAA GGA-3' and reverse, 5'-TGTTTCCATCTCACCAAATGC-3'; GAPDH forward, 5'-GCACCGTCAAGGCTGAGAAC-3' and reverse, 5'-TGGTGAAGACGCCAGTGGA-3'; miR-181b-5p forward, 5'-ACACTCCAGCTGGGACTTGGGCACTGA AACA-3' and reverse, 5'-TGGTGTCGTGGAGTCG-3' and U6 forward, 5'-CTCGCTTCGGCAGCACA-3' and reverse, 5'-AACGCTTCACGAATTTGCGT-3'. The thermocycling conditions were as follows: Initial denaturation at $95^{\circ} \mathrm{C}$ for $5 \mathrm{~min}$, then $95^{\circ} \mathrm{C}$ for $30 \mathrm{sec}, 60^{\circ} \mathrm{C}$ for $30 \mathrm{sec}$ and $72^{\circ} \mathrm{C}$ for $30 \mathrm{sec}$, for a total of 40 cycles. GAPDH and U6 served as the internal controls. Expression levels were quantified using the $2^{-\Delta \Delta \mathrm{Cq}}$ method (21). 
Western blotting. Following treatment, whole cell lysates were acquired using RIPA lysis buffer (Beyotime Institute of Biotechnology). Protein concentration was quantified using a bicinchoninic acid Protein Assay kit (Pierce; Thermo Fisher Scientific, Inc.) and $20 \mu \mathrm{g}$ protein was separated via $10 \%$ SDS-PAGE. The separated proteins were subsequently transferred onto nitrocellulose membranes and blocked in 5\% non-fat milk for $2 \mathrm{~h}$ at room temperature. The membranes were then incubated with the following primary antibodies at $4^{\circ} \mathrm{C}$ overnight: Anti-Bcl-2 (cat. no. ab32124; 1:1,000; Abcam), anti-Bax (cat. no. ab32503; 1:1,000; Abcam), anti-cleaved caspase-9 (cat. no. ab2324; 1:1,000; Abcam), anti-cleaved caspase-3 (cat. no. ab32042; 1:1,000; Abcam), anti-caspase 3 (cat. no. ab13847; 1:1,000; Abcam), anti-caspase-9 (cat. no. ab32539; 1:1,000; Abcam) and anti-GAPDH (cat. no. ab8245; 1:1,000; Abcam). Following primary antibody incubation, the membranes were incubated with horseradish peroxidase-conjugated secondary antibody (cat. no. 7074; 1:1,000; Cell Signaling Technology, Inc.) at room temperature for $2 \mathrm{~h}$ in the dark. Protein bands were visualized using an ECL Western Blotting Detection reagent (GE Healthcare) and densitometric analysis was performed using Image J software (v.1.52; National Institutes of Health).

ELISA. The cell supernatant was collected by centrifugation at $12,000 \mathrm{x} \mathrm{g}$ for $10 \mathrm{~min}$ at $4^{\circ} \mathrm{C}$ and the concentrations of inflammatory cytokines were measured using interleukin (IL)-1 $\beta$ ELISA kit (cat. no. PI305; Beyotime Institute of Biotechnology), IL-6 ELISA kit (cat. no. PI330; Beyotime Institute of Biotechnology) and tumor necrosis factor (TNF)- $\alpha$ kit (cat. no. PT518; Beyotime Institute of Biotechnology) according to the manufacturers' protocols. All experiments were repeated in triplicate.

Dual luciferase reporter assay. The combination between SNHG14 and miR-181b-5p were predicted using StarBase 3.0 (https://starbase.sysu.edu.cn/index.php). Cells were seeded into 24-well plates at a density of $5 \times 10^{4}$ cells/well and cultured at $37^{\circ} \mathrm{C}$ for $24 \mathrm{~h}$. The fragment of SNHG14 containing the predicted wild-type (WT) or mutant (MUT) miR-181b-5p-binding sequences were amplified by Shanghai GenePharma Co., Ltd., and inserted into the pmirGLO luciferase reporter gene vector (Promega Corporation) to produce the reporter plasmids SNHG14-WT and SNHG14-MUT, respectively. Subsequently, the cells were co-transfected with SNHG14-WT/SNHG14-MUT and miR-181b-5p mimic/mimic-NC using Lipofectamine 2000 reagent for $24 \mathrm{~h}$. The culture medium was then removed, and the cells were rinsed twice with PBS. The cells were added to the cell lysates, which were swirled for $10 \mathrm{~min}$ and centrifuged at $12,000 \mathrm{x} \mathrm{g}$ for $10 \mathrm{~min}$ at $4^{\circ} \mathrm{C}$, and the supernatant was subsequently transferred to a new Eppendorf tube. A dual luciferase kit (cat. no. D0010; Beijing Solarbio Science \& Technology Co., Ltd.) was used to measure luciferase activity according to the manufacturer's protocol. Firefly luminescence and Renilla luminescence were detected by enzyme markers, and Renilla luminescence was used as internal reference.

Statistical analysis. Each experiment was performed three times and data are presented as the mean \pm SD. Statistical analysis was performed using GraphPad Prism 5 software (GraphPad Software, Inc.). Statistical differences between groups were analyzed using one-way ANOVA with Tukey's test or Student's t-test. $\mathrm{P}<0.05$ was considered to indicate a statistically significant difference.

\section{Results}

LPS induces PC-12 cell inflammatory injury. CCK-8 assay was performed to determine the dose of LPS to use for further experiments. As presented in Fig. 1A, PC-12 cell viability was barely affected following treatment with $1 \mu \mathrm{g} / \mathrm{ml}$ LPS, whilst treatment with $5 \mu \mathrm{g} / \mathrm{ml}$ LPS significantly decreased the cell viability compared with the control group. Therefore, $5 \mu \mathrm{g} / \mathrm{ml}$ LPS was used for subsequent experiments. TUNEL assay results revealed a significant increase in cell apoptosis in LPS-treated cells compared with controls (Fig. 1B). These findings were validated using western blotting, which revealed that compared with controls, the expression levels of Bcl-2 were significantly downregulated, while the expression levels of Bax, cleaved caspase- 9 and cleaved caspase- 3 were significantly upregulated following treatment with $5 \mu \mathrm{g} / \mathrm{ml}$ LPS (Fig. 1C and D). As presented in Fig. 1E, the secretory levels of pro-inflammatory factors, including IL-1 $\beta$, IL-6 and TNF- $\alpha$, were significantly increased in the $5 \mu \mathrm{g} / \mathrm{ml}$ LPS group compared with controls. These results suggested that LPS could induce PC-12 cell inflammatory injury.

Knockdown of SNHG14 alleviates LPS-induced inflammation and apoptosis of PC-12 cells. RT-qPCR analysis revealed that the expression levels of SNHG14 in PC-12 cells were significantly upregulated following treatment with $5 \mu \mathrm{g} / \mathrm{ml}$ LPS compared with controls (Fig. 2A). After constructing the interference plasmids and transfecting them into PC-12 cells, shRNA-SNHG14-1 demonstrated greater transfection efficiency compared with shRNA-SNHG14-2. Therefore, shRNA-SNHG14-1 was selected for use in subsequent experiments (Fig. 2B). PC-12 cells treated with LPS and transfected with shRNA-SNHG14 had significantly higher cell viability (Fig. 2C) and significantly lower cell apoptosis levels (Fig. 2D) compared with those treated with LPS alone. In addition, western blotting results revealed that the expression levels of Bcl-2 were significantly upregulated, while the expression levels of Bax, cleaved caspase- 9 and cleaved caspase-3 were significantly downregulated in cells treated with $5 \mu \mathrm{g} / \mathrm{ml}$ LPS and transfected with shRNA-SNHG14 compared with the $5 \mu \mathrm{g} / \mathrm{ml}$ LPS group (Fig. 2E and F). ELISA results illustrated that the secretory levels of IL-1 $\beta$, IL-6 and TNF- $\alpha$ in PC-12 cells following LPS treatment were significantly downregulated when the cells were also transfected with shRNA-SNHG14 (Fig. 2G). These results suggested SNHG14 knockdown may alleviate LPS-induced inflammation and apoptosis of PC-12 cells.

SNHG14 negatively regulates miR-181b-5p expression levels in PC-12 cells. To investigate the regulatory mechanism of SNHG14 in PC-12 cells, RT-qPCR analysis was conducted to determine the interaction between SNHG14 and miR-181b-5p. As presented in Fig. 3A, compared with the control, the mRNA expression levels of miR-181b-5p were significantly 


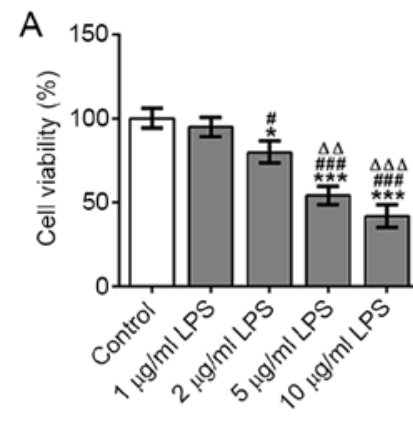

C

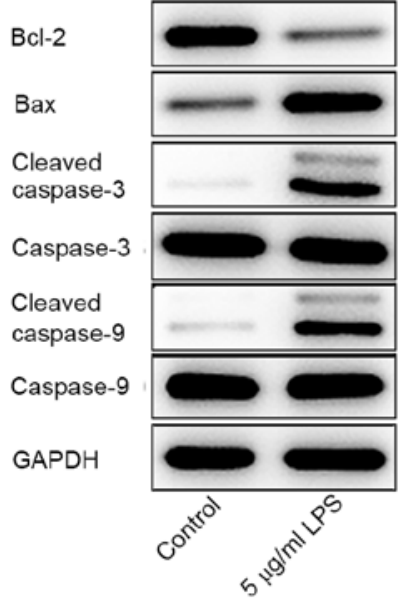

B

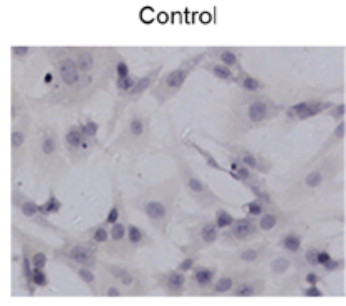

D
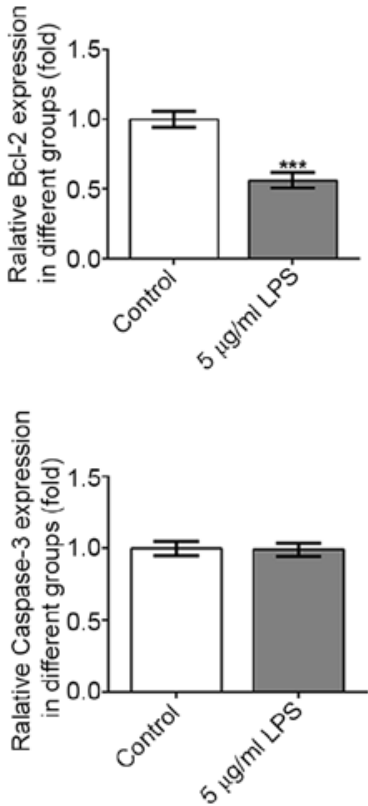

E

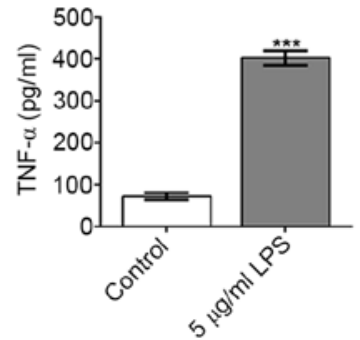

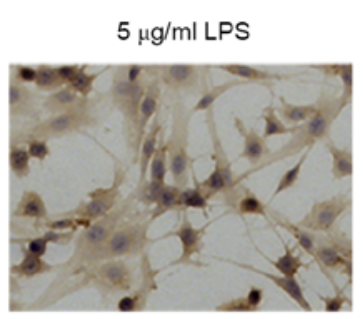
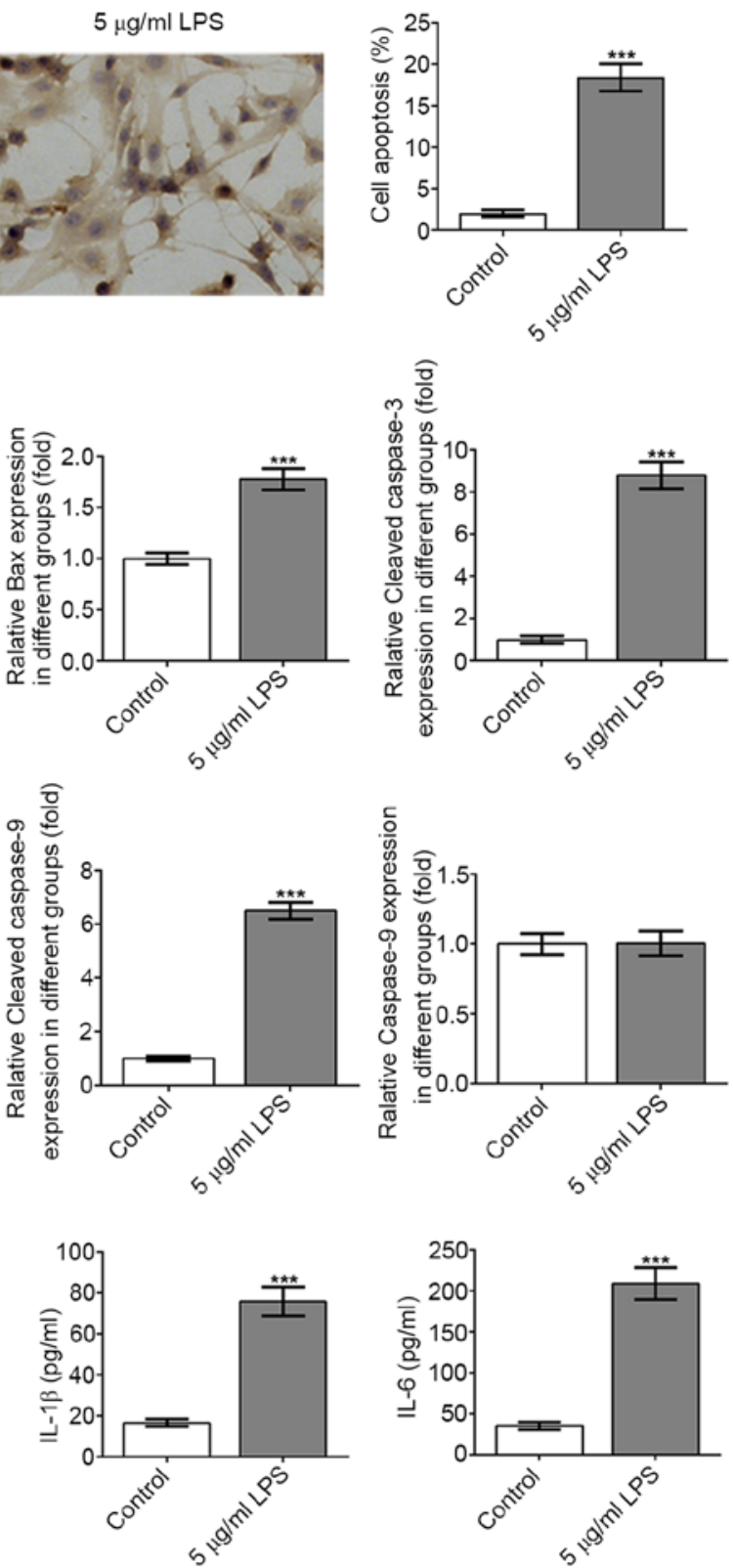

Figure 1. LPS induces the apoptosis of PC-12 cells. (A) PC-12 cell viability after LPS induction at different concentrations was detected by Cell Counting Kit- 8 assay. ${ }^{~} \mathrm{P}<0.05$ and ${ }^{* * * *} \mathrm{P}<0.001$ vs. Control group. ${ }^{*} \mathrm{P}<0.05$ and ${ }^{\# \# \#} \mathrm{P}<0.001$ vs. $1 \mu \mathrm{g} / \mathrm{ml}$ LPS group. ${ }^{\Delta \Delta} \mathrm{P}<0.01$ and ${ }^{\Delta \Delta}{ }^{\Delta} \mathrm{P}<0.001$ vs. $2 \mu \mathrm{g} / \mathrm{ml}$ LPS group. (B) PC-12 cell apoptosis after LPS induction was detected by TUNEL assay. Magnification, $\mathrm{x} 200$. ${ }^{* * *} \mathrm{P}<0.001 \mathrm{vs}$. Control group. (C) The expression of apoptosis-related proteins was analyzed by western blot analysis and (D) quantified. ${ }^{* * *} \mathrm{P}<0.001 \mathrm{vs.} \mathrm{Control} \mathrm{group.} \mathrm{(E)} \mathrm{The} \mathrm{levels} \mathrm{of} \mathrm{TNF-} \alpha$, IL-1 $1 \beta$ and IL- 6 in the supernatant of PC-12 cells after LPS induction were detected by ELISA. ${ }^{* * *} \mathrm{P}<0.001$ vs. Control group. LPS, lipopolysaccharide.

downregulated following treatment with $5 \mu \mathrm{g} / \mathrm{ml}$ LPS. However, the expression levels of miR-181b-5p in PC-12 cells were significantly upregulated following SNHG14 knockdown compared with the control group (Fig. 3B). Subsequently, a miR-181b-5p mimic was constructed and successfully transfected into PC-12 cells to overexpress miR-181b-5p, as presented in Fig. 3C. The expression levels of SNHG14 were significantly downregulated in PC-12 cells transfected with miR-181b-5p mimic compared with the control (Fig. 3D). The binding sites between SNHG14 and miR-181b-5p were subsequently predicted (Fig. 3E). Dual luciferase reporter assay results revealed that the relative luciferase activities of PC-12 cells did not change following co-transfection with mimic-NC + SNHG14-MUT or miR-181b-5p mimic + SNHG14-MUT; however, the relativeluciferaseactivity wassignif- icantly decreased following co-transfection with miR-181b-5p mimic + SNHG14-WT (Fig. 3F). These findings indicated that SNHG14 may negatively regulate miR-181b-5p in PC-12 cells.

Knockdown of SNHG14 alleviates LPS-induced inflammation and apoptosis of PC-12 cells via upregulating miR-181b-5p expression levels. To confirm whether SNHG14 could reverse inflammation and apoptosis in PC-12 cells through the newly identified regulatory mechanism, a CCK-8 assay was performed. The results revealed that the decreased cell viability induced by LPS stimulation was rescued following transfection with shRNA-SNHG14, while the addition of the miR-181b-5p inhibitor significantly decreased cell viability (Fig. 4A). In addition, as presented in Fig. 4B, cell apoptosis was 
A

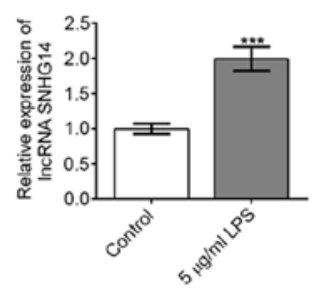

B

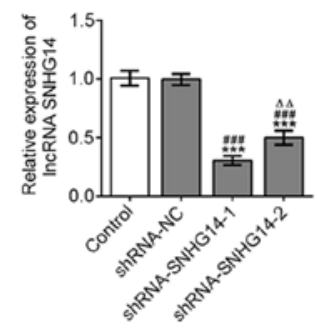

C

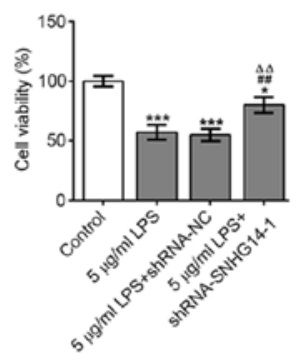

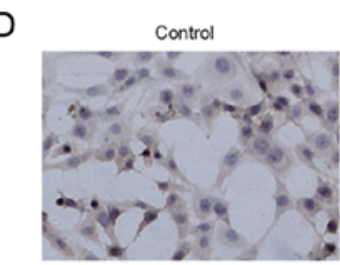

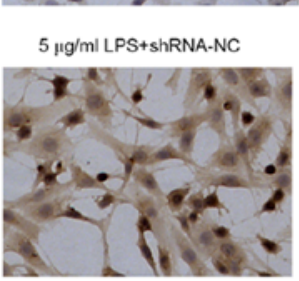

$\mathrm{F}$
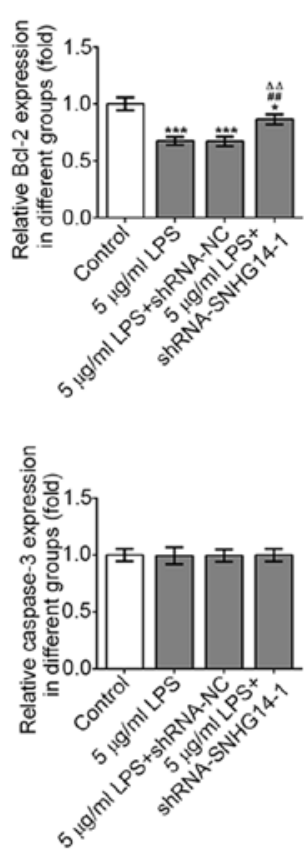

G

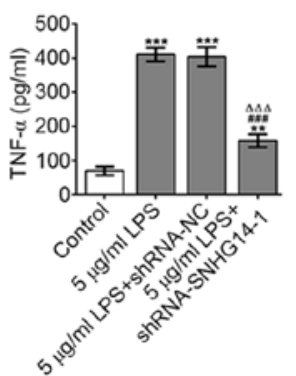

$5 \mu \mathrm{g} / \mathrm{ml}$ LPS
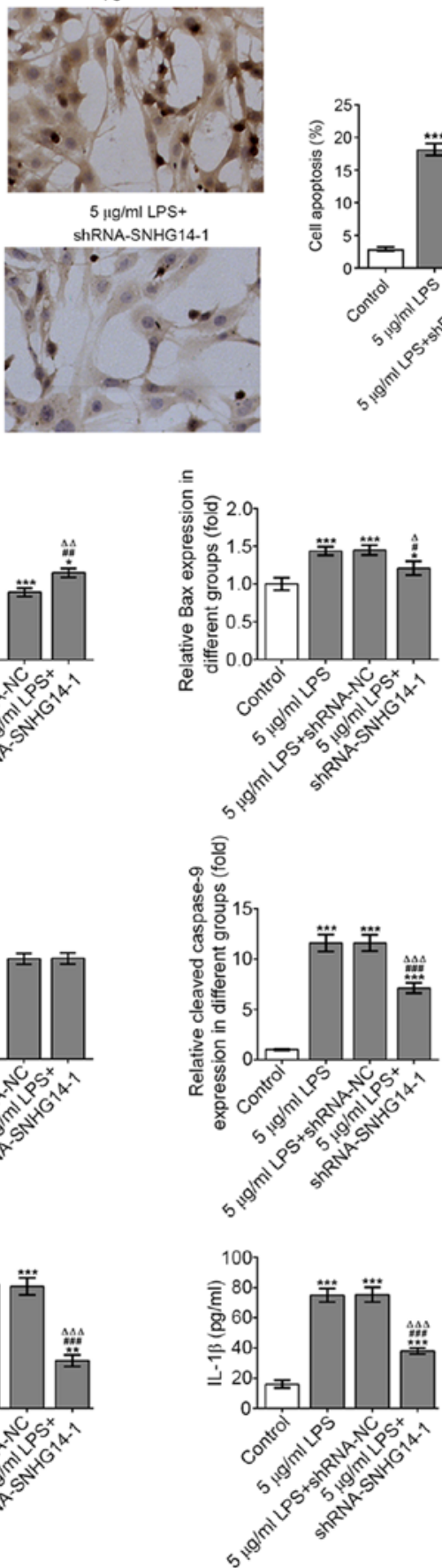

$\mathrm{E}$

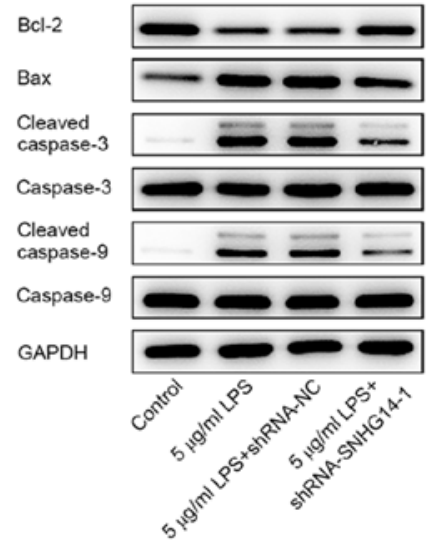

$\mathrm{Bcl}-2$

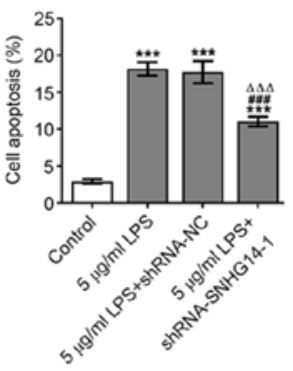

caspase-3

Caspase-3

aspase-9

Caspase- 9
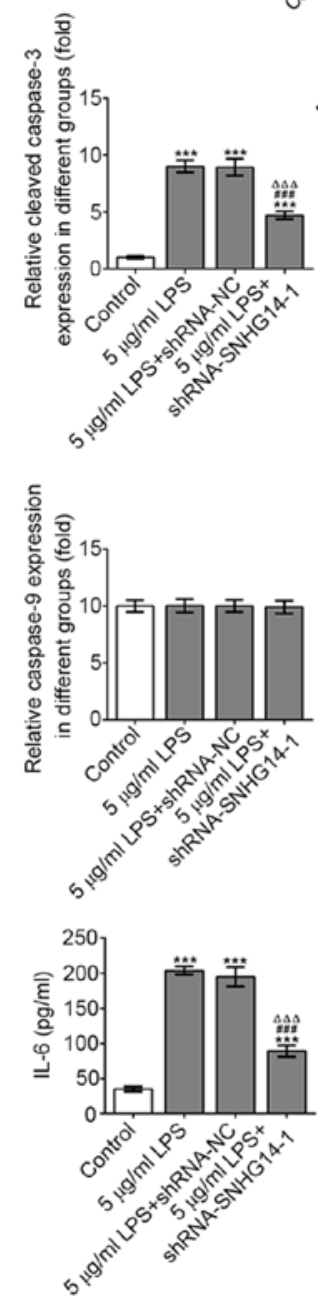

Figure 2. SNHG14 knockdown alleviates inflammation and apoptosis of PC-12 cells induced by LPS. (A) SNHG14 expression in PC-12 cells after LPS induction was detected by RT-qPCR. ${ }^{* * * *} \mathrm{P}<0.001$ vs. Control group. (B) The transfection efficiency of shRNA-SNHG14-1/2 was verified by RT-qPCR. ${ }^{* * * *} \mathrm{P}<0.001$ vs. Control group. ${ }^{\# \# "} \mathrm{P}<0.001$ vs. shRNA-NC group. ${ }^{\Delta \Delta} \mathrm{P}<0.01$ vs. shRNA-SNHG14-1 group. (C) LPS-induced PC12 cell viability after transfection was detected by Cell Counting Kit-8 assay. ${ }^{*} \mathrm{P}<0.05$ and ${ }^{* * *} \mathrm{P}<0.001$ vs. Control group. ${ }^{\# \#} \mathrm{P}<0.01$ vs. $5 \mu \mathrm{g} / \mathrm{ml}$ LPS group. ${ }^{\Delta \Delta} \mathrm{P}<0.01$ vs. $5 \mu \mathrm{g} / \mathrm{ml}$ LPS + shRNA-NC group. (D) LPS-induced PC12 cell apoptosis after transfection was detected by TUNEL assay. Magnification, $\mathrm{x} 200 .{ }^{* * *} \mathrm{P}<0.001$ vs. Control group. ${ }^{\# \# *} \mathrm{P}<0.001$ vs. $5 \mu \mathrm{g} / \mathrm{ml}$ LPS group. ${ }^{\Delta \Delta \Delta} \mathrm{P}<0.001 \mathrm{vs} .5 \mu \mathrm{g} / \mathrm{ml}$ LPS + shRNA-NC group. (E) The expression of apoptosis-related proteins was analyzed by western blot analysis and (F) quantified. ${ }^{*} \mathrm{P}<0.05$ and ${ }^{* * * *} \mathrm{P}<0.001$ vs. Control group. ${ }^{\#} \mathrm{P}<0.05,{ }^{\# \#} \mathrm{P}<0.01$ and ${ }^{\# \# \prime} \mathrm{P}<0.001$ vs. $5 \mu \mathrm{g} / \mathrm{ml}$ LPS group. ${ }^{\Delta} \mathrm{P}<0.05,{ }^{\Delta \Delta} \mathrm{P}<0.01$ and ${ }^{\Delta \Delta \Delta} \mathrm{P}<0.001$ vs. $5 \mu \mathrm{g} / \mathrm{ml}$ LPS + shRNA-NC group. (G) The levels of TNF- $\alpha$, IL-1 $\beta$ and IL-6 in the supernatant of LPS-induced PC12 cells after transfection were detected by ELISA. ${ }^{* *} \mathrm{P}<0.01$ and ${ }^{* * *} \mathrm{P}<0.001$ vs. Control group. ${ }^{\# \# \#} \mathrm{P}<0.001$ vs. $5 \mu \mathrm{g} / \mathrm{ml}$ LPS group. ${ }^{\Delta \Delta}{ }^{\Delta} \mathrm{P}<0.001$ vs. $5 \mu \mathrm{g} / \mathrm{ml}$ LPS + shRNA-NC group. SNHG14, small nucleolar host gene 14; LPS, lipopolysaccharide; shRNA, short hairpin RNA; NC, negative control; RT-qPCR, reverse transcription-quantitative PCR. 
A

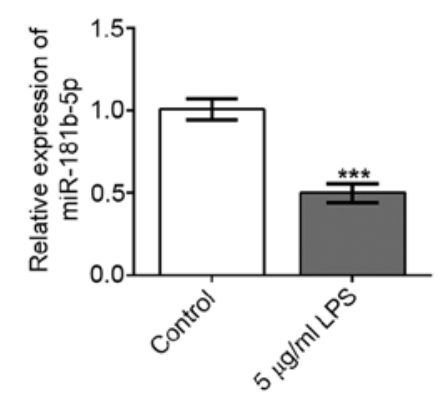

D

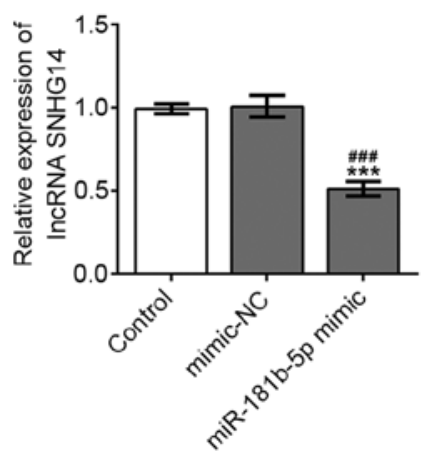

B

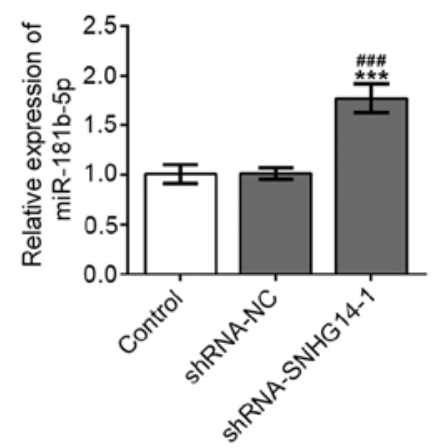

C

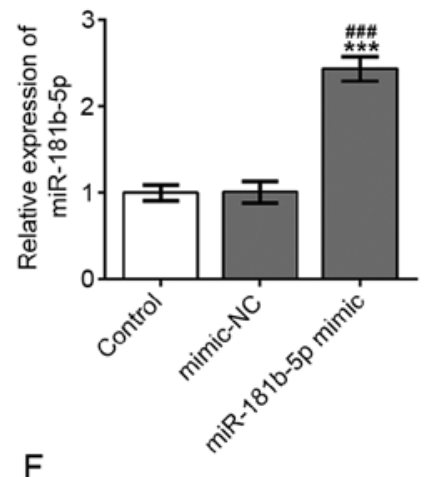

$\mathrm{F}$

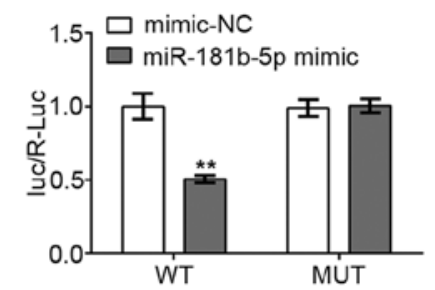

Figure 3. SNHG14 negatively regulates miR-181b-5p in PC-12 cells. (A) miR-181b-5p expression in PC12 cells after LPS induction was detected by RT-qPCR ${ }_{* * * * *} \mathrm{P}<0.001$ vs. Control group. (B) miR-181b-5p expression in PC12 cells transfected with shRNA-SNHG14-1 was detected by RT-qPCR. ${ }^{* * * * *} \mathrm{P}<0.001 \mathrm{vs}$. Control group. ${ }^{\# \# \# ~} \mathrm{P}<0.001$ vs. shRNA-NC group. (C) The transfection efficiency of miR-181b-5p mimic was verified by RT-qPCR. ${ }^{* * *} \mathrm{P}<0.001$ vs. Control group.

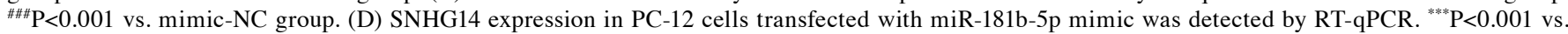
Control group. ${ }^{\# \# \#} \mathrm{P}<0.001$ vs. mimic-NC group. (E) Binding sites between SNHG14 and miR-181b-5p. (F) Luciferase reporter assay verified the direct binding relationship between SNHG14 and miR-181b-5p. ${ }^{* *} \mathrm{P}<0.01$ vs. mimic-NC group. SNHG14, small nucleolar host gene 14; LPS, lipopolysaccharide; shRNA, short hairpin RNA; NC, negative control; RT-qPCR, reverse transcription-quantitative PCR; miR, microRNA; WT, wild-type; MUT, mutant; luc, luciferase; R-Luc, Renilla luciferase; lncRNA, long non-coding RNA.

significantly decreased following stimulation with LPS and transfection with shRNA-SNHG14, while co-transfection with shRNA-SNHG14 and miR-181b-5p inhibitor into LPS-treated cells attenuated cell apoptosis. The downregulated expression levels of Bcl-2 and upregulated expression levels of Bax, cleaved caspase- 9 and cleaved caspase- 3 induced by $5 \mu \mathrm{g} / \mathrm{ml}$ LPS were reversed by SNHG14 knockdown (Fig. 4C and D). However, miR-181b-5p inhibition suppressed the effects of SNHG14 knockdown in LPS-induced cells. As presented in Fig. 4E, the secretory levels of proinflammatory factors IL-1 $\beta$, IL-6 and TNF- $\alpha$ were downregulated following stimulation with LPS and transfection with shRNA-SNHG14. However, following LPS stimulation, the secretory levels of these inflammatory factors were increased when the cells were co-transfected with shRNA-SNHG14 and miR-181b-5p inhibitor compared with those transfected with shRNA-SNHG14 alone. These results suggested that SNHG14 knockdown may upregulate the expression levels of miR-181b-5p and subsequently attenuate LPS-induced inflammation and apoptosis of PC-12 cells.

\section{Discussion}

Despite progress being made in understanding the basic neurobiology of SCI, strategies available for the treatment of SCI remain limited (22). Current clinical treatments, including surgical procedures and high-dose methylprednisolone, can improve the overall survival rate of patients, but they cannot repair nerve function that has been damaged (23). In the present study, the role of IncRNA SNHG14 in LPS-induced PC-12 cells was investigated, in addition to its potential mechanism, with the aim to provide a novel strategy for the treatment of SCI.

IncRNAs serve a role in various fundamental biochemical and cellular processes and have emerged as pivotal regulators that can enhance neural regeneration in the development of SCI (24). Moreover, the expression levels of certain lncRNAs, such as SNHG5 and tectonic family member 2, have been discovered to be upregulated in SCI $(25,26)$. Therefore, therapies that target lncRNAs may hold promise for the treatment of SCI (27). SNHG14 is located within the Prader-Willi critical region and extends into the region of the ubiquitin protein ligase E3A gene, whose deficiency in brain cells in children was revealed to contribute to neurogenetic disorders (28). A previous study has verified that SNHG14 may aggravate inflammation in cerebral ischemia/reperfusion injury by damaging the miR-136-5p-dependent inhibition of ROCK1 (15). It is well known that inflammation is one of the most common features in the occurrence of the SCI (29). Therefore, it was hypothesized that SNHG14 may also induce the development of SCI. In the present study, inflammatory injury following SCI was stimulated in cells by LPS treatment, and the results revealed that the expression levels of SNHG14 were upregulated following LPS stimulation compared with the control group. Following the subsequent construction of interference plasmids targeting SNHG14 and transfection into PC-12 cells, 
A

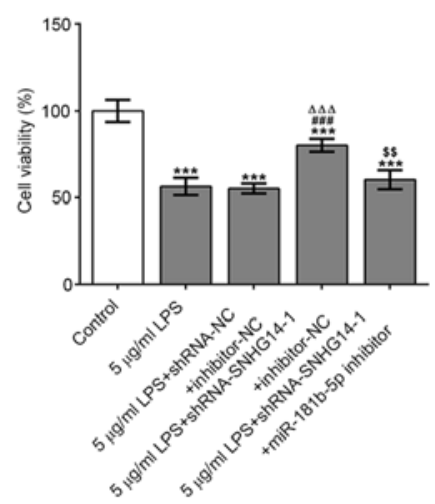

C

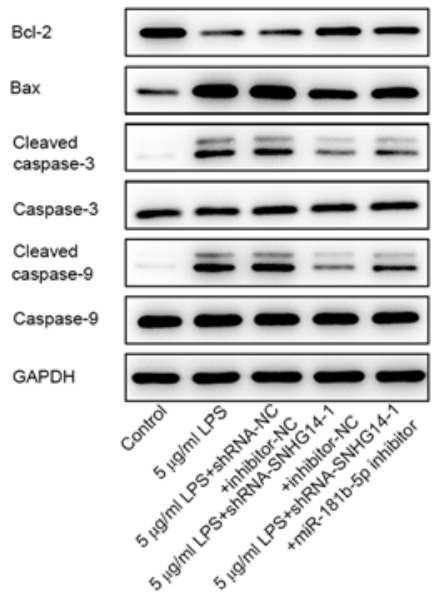

B

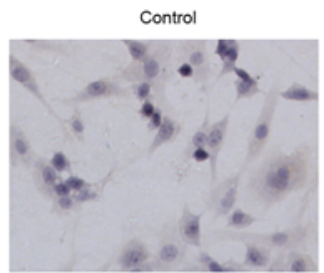

$5 \mu \mathrm{g} / \mathrm{ml}$ LPS+shRNA-SNHG14-1 tinhibitor-NC

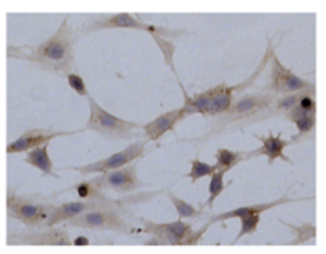

$5 \mu \mathrm{g} / \mathrm{ml}$ LPS

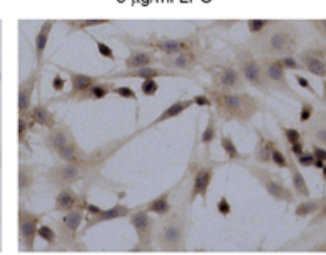

$\mu \mathrm{g} / \mathrm{ml}$ LPS+shRNA-SNHG14 + miR-181b-5p inhibitor

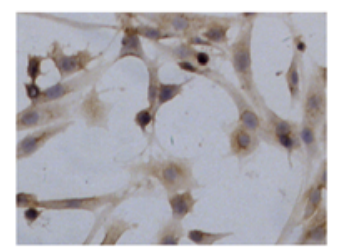

$5 \mu / \mathrm{ml}$ LPS+shRNA-NC+inhibitor-NC
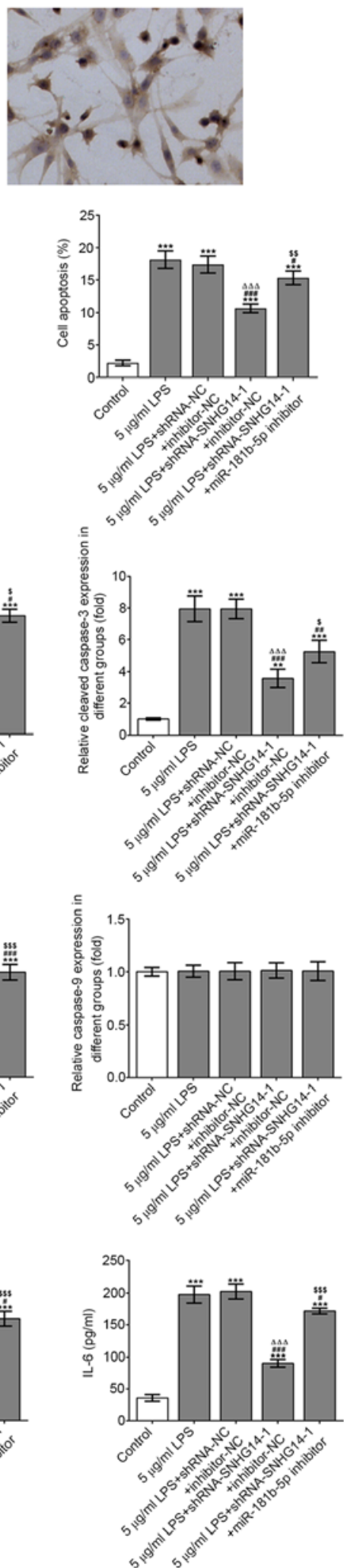

Figure 4. SNHG14 knockdown alleviates inflammation and apoptosis of PC-12 cells induced by LPS via upregulating miR-181b-5p. (A) LPS-induced PC12 cell viability after transfection was detected by Cell Counting Kit- 8 assay. ${ }^{* * *} \mathrm{P}<0.001 \mathrm{vs}$. Control group. ${ }^{\# \# t} \mathrm{P}<0.001 \mathrm{vs} .5 \mu \mathrm{g} / \mathrm{ml}$ LPS group. ${ }^{\Delta \Delta \Delta} \mathrm{P}<0.001 \mathrm{vs} .5 \mu \mathrm{g} / \mathrm{ml}$ LPS + shRNA-NC + inhibitor-NC group. ${ }^{\$ \$} \mathrm{P}<0.01$ vs. $5 \mu \mathrm{g} / \mathrm{ml}$ LPS + shRNA-SNHG14-1 + inhibitor-NC group. (B) LPS-induced PC12 cell apoptosis after transfection was detected by TUNEL assay. Magnification, $\mathrm{x} 200 .{ }^{* * * *} \mathrm{P}<0.001$ vs. Control group. ${ }^{*} \mathrm{P}<0.05$ and ${ }^{\# \# \#} \mathrm{P}<0.001 \mathrm{vs} .5 \mu \mathrm{g} / \mathrm{ml} \mathrm{LPS}$ group. ${ }^{\Delta \Delta \Delta} \mathrm{P}<0.001 \mathrm{vs}$. $5 \mu \mathrm{g} / \mathrm{ml}$ LPS + shRNA-NC + inhibitor-NC group. ${ }^{\$ \$} \mathrm{P}<0.01$ vs. $5 \mu \mathrm{g} / \mathrm{ml}$ LPS + shRNA-SNHG14-1 + inhibitor-NC group. (C) The expression of apoptosis-related proteins was analyzed by western blot analysis and (D) quantified. ${ }^{* *} \mathrm{P}<0.01$ and ${ }^{* * * *} \mathrm{P}<0.001$ vs. Control group. ${ }^{\#} \mathrm{P}<0.05,{ }^{\# \#} \mathrm{P}<0.01$ and ${ }^{\# \# \#} \mathrm{P}<0.001 \mathrm{vs} .5 \mu \mathrm{g} / \mathrm{ml}$ LPS group. ${ }^{\Delta \Delta} \mathrm{P}<0.01$ and ${ }^{\Delta \Delta \Delta} \mathrm{P}<0.001$ vs. $5 \mu \mathrm{g} / \mathrm{ml}$ LPS + shRNA-NC + inhibitor-NC group. ${ }^{\$} \mathrm{P}<0.05$ and ${ }^{\$ \$ \$} \mathrm{P}<0.001$ vs. $5 \mu \mathrm{g} / \mathrm{ml}$ LPS + shRNA-SNHG14-1 + inhibitor-NC group. (E) The levels of TNF- $\alpha$, IL-1 $\beta$ and IL- 6 in the supernatant of LPS-induced PC12 cells after transfection were detected by ELISA. ${ }^{* *} \mathrm{P}<0.01$ and ${ }^{* * *} \mathrm{P}<0.001$ vs. Control group. ${ }^{*} \mathrm{P}<0.05,{ }^{\# \prime} \mathrm{P}<0.01$ and ${ }^{\# \# "} \mathrm{P}<0.001$ vs. $5 \mu \mathrm{g} / \mathrm{ml}$ LPS group. ${ }^{\Delta \Delta \Delta} \mathrm{P}<0.001$ vs. $5 \mu \mathrm{g} / \mathrm{ml}$ LPS + shRNA-NC + inhibitor-NC group. ${ }^{\$ \$ S} \mathrm{P}<0.001$ vs. $5 \mu \mathrm{g} / \mathrm{ml}$ LPS + shRNA-SNHG14-1 + inhibitor-NC group. SNHG14, small nucleolar host gene 14; LPS, lipopolysaccharide; shRNA, short hairpin RNA; NC, negative control; miR, microRNA; WT, wild-type; MUT, mutant; luc, luciferase; lncRNA, long non-coding RNA. 
it was observed that SNHG14 knockdown could rescue cell viability under an LPS-induced inflammatory environment. Since the sub-acute phase of secondary SCI is involved in apoptosis, the expression levels of apoptosis-related proteins in PC-12 cells were analyzed (30). The results revealed that the genetic silencing of SNHG14 could reduce cell apoptosis and alleviate inflammation. These findings suggested that SNHG14 may facilitate the progression of SCI, which was consistent with the findings of Jiao et al (31), who reported that SNHG14 participated in the deterioration of non-small cell lung cancer.

Using advanced high-throughput sequencing technologies, a large number of differentially expressed genes (lncRNA LINRIS, IncRNA HOXA-AS2 and lncRNA ZNF667-AS1), which participated in the development of various types of disease, have been identified (32-34). Previous research has also noted the role of miRNAs following SCI and suggested that they could be novel biomarkers for the diagnosis, treatment and prognosis of such injuries (35). Accumulating evidence has verified that miRNAs can regulate their expression and subsequent biological functions by interacting with lncRNAs (36). LncRNAs have been found to exert their effects by targeting or sponging miRNAs in various types of cancer (37-40). However, whether lncRNAs could sponge or target a certain miRNA in SCI remain to be determined. The present study used StarBase 3.0 software to predict that miR-181b-5p, a member of the miR-181 family, was a target of SNHG14. Indeed, the genetic knockdown of SNHG14 upregulated miR-181b-5p expression levels, while SNHG14 expression levels in PC-12 cells transfected with a miR-181b-5p mimic were downregulated, indicating the negative regulatory role of SNHG14 on miR-181b-5p expression levels. Furthermore, miR-181b-5p was discovered to promote cell viability and inhibit the apoptosis of PC-12 cells.

In conclusion, the results of the present study suggested that SNHG14 may serve as a pathogenic lncRNA in PC-12 cells, as the genetic knockdown of SNHG14 alleviated LPS-induced inflammation and apoptosis of PC-12 cells by negatively regulating miR-181b-5p expression levels. These results may provide a novel perspective for future therapeutic strategies for SCI. However, the present study is that only performed in vitro experiments. Future studies will involve in vivo experiments and assess other underlying mechanisms.

\section{Acknowledgements}

Not applicable.

\section{Funding}

No funding was received.

\section{Availability of data and materials}

The datasets used and/or analyzed during the current study are available from the corresponding author on reasonable request.

\section{Authors' contributions}

YX conceived and designed the experiments. HJ and JN performed all the experiments and $\mathrm{YZ}$ helped $\mathrm{HJ}$ and $\mathrm{JN}$ to collect experimental data. $\mathrm{HJ}$ and $\mathrm{JN}$ authenticated the raw data in this study. YZ performed the statistical analysis. $\mathrm{HJ}$ and JN wrote the paper together which was revised and polished by YX. All authors reviewed and approved the final manuscript.

\section{Ethics approval and consent to participate}

Not applicable.

\section{Patient consent for publication}

Not applicable.

\section{Competing interests}

The authors declare that they have no competing interests.

\section{References}

1. Lu Y, Yang J, Wang X, Ma Z, Li S, Liu Z and Fan X: Research progress in use of traditional Chinese medicine for treatment of spinal cord injury. Biomed Pharmacother 127: 110136, 2020.

2. Watanabe S, Uchida K, Nakajima H, Matsuo H, Sugita D, Yoshida A, Honjoh K, Johnson WE and Baba H: Early transplantation of mesenchymal stem cells after spinal cord injury relieves pain hypersensitivity through suppression of pain-related signaling cascades and reduced inflammatory cell recruitment. Stem Cells 33: 1902-1914, 2015.

3. Hamann K, Nehrt G, Ouyang H, Duerstock B and Shi R: Hydralazine inhibits compression and acrolein-mediated injuries in ex vivo spinal cord. J Neurochem 104: 708-718, 2008.

4. Hamann K and Shi R: Acrolein scavenging: A potential novel mechanism of attenuating oxidative stress following spinal cord injury. J Neurochem 111: 1348-1356, 2009.

5. Saxena T, Loomis KH, Pai SB, Karumbaiah L, Gaupp E, Patil K, Patkar R and Bellamkonda RV: Nanocarrier-mediated inhibition of macrophage migration inhibitory factor attenuates secondary injury after spinal cord injury. ACS Nano 9: 1492-1505, 2015

6. Lv ZC, Cao XY, Guo YX, Zhang XD, Ding J, Geng J, Feng K and Niu H: MiR-137-5p alleviates inflammation by upregulating IL-10R1 expression in rats with spinal cord injury. Eur Rev Med Pharmacol Sci 23: 4551-4557, 2019.

7. Zhu Y,Zhu H, Wang Z, Gao F, Wang J and Zhang W: Wogonoside alleviates inflammation induced by traumatic spinal cord injury by suppressing NF- $\kappa \mathrm{B}$ and NLRP3 inflammasome activation. Exp Ther Med 14: 3304-3308, 2017.

8. Iyer MK, Niknafs YS, Malik R, Singhal U, Sahu A, Hosono Y, Barrette TR, Prensner JR, Evans JR, Zhao S, et al: The landscape of long noncoding RNAs in the human transcriptome. Nat Genet 47: 199-208, 2015.

9. Zhang Y, Xia Q and Lin J: LncRNA H19 Attenuates apoptosis in MPTP-induced Parkinson's disease through regulating miR-585-3p/PIK3R3. Neurochem Res 45: 1700-1710, 2020.

10. Gao J, Wang F, Wu P, Chen Y and Jia Y: Aberrant lncRNA expression in leukemia. J Cancer 11: 4284-4296, 2020.

11. Zhu W, Liu $\mathrm{H}$, Wang $\mathrm{X}$, Lu J and Yang W: Long noncoding RNAs in bladder cancer prognosis: A meta-analysis. Pathol Res Pract 215: 152429, 2019.

12. Loewen G, Jayawickramarajah J, Zhuo Y and Shan B: Functions of lncRNA HOTAIR in lung cancer. J Hematol Oncol 7: 90, 2014.

13. Liu Y, Liu C, Zhang A, Yin S, Wang T, Wang Y, Wang M, Liu Y, Ying Q, Sun J, et al: Down-regulation of long non-coding RNA MEG3 suppresses osteogenic differentiation of periodontal ligament stem cells (PDLSCs) through miR-27a-3p/IGF1 ax is in periodontitis. Aging (Albany NY) 11: 5334-5350, 2019.

14. Li Z, Ho IHT, Li X, Xu D, Wu WKK, Chan MTV, Li S and Liu X: Long non-coding RNAs in the spinal cord injury: Novel spotlight. J Cell Mol Med 23: 4883-4890, 2019.

15. Zhong Y, Yu C and Qin W: LncRNA SNHG14 promotes inflammatory response induced by cerebral ischemia/reperfusion injury through regulating miR-136-5p/ROCK1. Cancer Gene Ther 26: 234-247, 2019. 
16. Zhu J, Bai J, Wang S and Dong H: Down-regulation of long noncoding RNA SNHG14 protects against acute lung injury induced by lipopolysaccharide through microRNA-34c-3p-dependent inhibition of WISP1. Respir Res 20: 233, 2019.

17. Bartel DP: MicroRNAs: Genomics, biogenesis, mechanism, and function. Cell 116: 281-297, 2004.

18. Ambros V: The functions of animal microRNAs. Nature 431 350-355, 2004.

19. Wang X, Sun H, Liu H, Ma L, Jiang C, Liao H, Xu S, Xiang J and Cao Z: MicroRNA-181b-5p modulates tumor necrosis factor$\alpha$-induced inflammatory responses by targeting interleukin- 6 in cementoblasts. J Cell Physiol 234: 22719-22730, 2019.

20. Zhao H, Guo Y, Sun Y, Zhang N and Wang X: miR-181a/b-5p ameliorates inflammatory response in monocrotaline-induced pulmonary arterial hypertension by targeting endocan. J Cell Physiol 235: 4422-4433, 2020.

21. Livak KJ and Schmittgen TD: Analysis of relative gene expression data using real-time quantitative PCR and the 2(-Delta Delta C(T)) method. Methods 25: 402-408, 2001.

22. Demirel A, Li J, Morrow C, Barnes S, Jansen J, Gower B, Kirksey K, Redden D and Yarar-Fisher C: Evaluation of a ketogenic diet for improvement of neurological recovery in individuals with acute spinal cord injury: Study protocol for a randomized controlled trial. Trials 21: 372, 2020.

23. Haldrup M, Schwartz OS, Kasch $\mathrm{H}$ and Rasmussen MM: Early decompressive surgery in patients with traumatic spinal cord injury improves neurological outcome. Acta Neurochir (Wien) 161: 2223-2228, 2019.

24. Zhu S, Zhou Z, Li Z, Shao J, Jiao G, Huang YE and Lin Y: Suppression of LINC00707 alleviates lipopolysaccharideinduced inflammation and apoptosis in PC-12 cells by regulated miR-30a-5p/Neurod 1. Biosci Biotechnol Biochem 83 : 2049-2056, 2019.

25. Wang F, Liu J, Wang X, Chen J, Kong Q, Ye B and Li Z: The emerging role of lncRNAs in spinal cord injury. Biomed Res Int 2019: 3467121, 2019.

26. Ren XD, Wan CX and Niu YL: Overexpression of lncRNA TCTN2 protects neurons from apoptosis by enhancing cell autophagy in spinal cord injury. FEBS Open Bio 9: 1223-1231, 2019.

27. Sun X, Shen H, Liu S, Gao J and Zhang S: Long noncoding RNA SNHG14 promotes the aggressiveness of retinoblastoma by sponging microRNA124 and thereby upregulating STAT3. Int J Mol Med 45: 1685-1696, 2020.

28. Qi X, Shao M, Sun H, Shen Y, Meng D and Huo W: Long non-coding RNA SNHG14 promotes microglia activation by regulating miR-145-5p/PLA2G4A in cerebral infarction. Neuroscience 348: 98-106, 2017.
29. Venkatesh K, Ghosh SK, Mullick M, Manivasagam G and Sen D: Spinal cord injury: Pathophysiology, treatment strategies, associated challenges, and future implications. Cell Tissue Res 377: 125-151, 2019.

30. Alizadeh A, Dyck SM and Karimi-Abdolrezaee S: Traumatic spinal cord injury: An overview of pathophysiology, models and acute injury mechanisms. Front Neurol 10: 282, 2019.

31. Jiao P, Hou J, Yao M, Wu J and Ren G: SNHG14 silencing suppresses the progression and promotes cisplatin sensitivity in non-small cell lung cancer. Biomed Pharmacother 117: 109164, 2019.

32. Wang Y, Lu JH, Wu QN, Jin Y, Wang DS, Chen YX, Liu J, Luo XJ, Meng Q, Pu HY, et al: LncRNA LINRIS stabilizes IGF2BP2 and promotes the aerobic glycolysis in colorectal cancer. Mol Cancer 18: 174, 2019.

33. Wang J, Su Z, Lu S, Fu W, Liu Z, Jiang X and Tai S: LncRNA HOXA-AS2 and its molecular mechanisms in human cancer. Clin Chim Acta 485: 229-233, 2018.

34. Li JW, Kuang Y, Chen L and Wang JF: LncRNA ZNF667-AS1 inhibits inflammatory response and promotes recovery of spinal cord injury via suppressing JAK-STAT pathway. Eur Rev Med Pharmacol Sci 22: 7614-7620, 2018.

35. Li F and Zhou MW: MicroRNAs in contusion spinal cord injury: Pathophysiology and clinical utility. Acta Neurol Belg 119: 21-27, 2019.

36. Wang Q, Teng Y, Wang R, Deng D, You Y, Peng Y, Shao N and Zhi F: The long non-coding RNA SNHG14 inhibits cell proliferation and invasion and promotes apoptosis by sponging miR-92a-3p in glioma. Oncotarget 9: 12112-12124, 2018.

37. Liang Y, Song X, Li Y, Chen B, Zhao W, Wang L, Zhang H, Liu Y, Han D, Zhang N, et al: LncRNA BCRT1 promotes breast cancer progression by targeting miR-1303/PTBP3 axis. Mol Cancer 19: 85, 2020.

38. Zhao W, Geng D, Li S, Chen Z and Sun M: LncRNA HOTAIR influences cell growth, migration, invasion, and apoptosis via the miR-20a-5p/HMGA2 axis in breast cancer. Cancer Med 7: 842-855, 2018.

39. Luan X and Wang Y: LncRNA XLOC_006390 facilitates cervical cancer tumorigenesis and metastasis as a ceRNA against miR-331-3p and miR-338-3p. J Gynecol Oncol 29: e95, 2018.

40. Hu YP, Jin YP, Wu XS, Yang Y, Li YS, Li HF, Xiang SS, Song XL, Jiang L, Zhang YJ, et al: LncRNA-HGBC stabilized by HuR promotes gallbladder cancer progression by regulating miR-502-3p/SET/AKT axis. Mol Cancer 18: 167, 2019.

This work is licensed under a Creative Commons Attribution-NonCommercial-NoDerivatives 4.0 International (CC BY-NC-ND 4.0) License. 